\section{A comparison of visual performance between a rigid PMMA and a foldable acrylic intraocular lens}

\section{Abstract}

Purpose To examine post-operative visual outcomes when using micro-incision surgery and a three-piece, foldable acrylic intraocular lens (IOL) and when using small-incision surgery and a one-piece, rigid polymethylmethacrylate (PMMA) IOL.

Methods Phacoemulsification and IOL implantation was conducted by one surgeon on 86 subjects (aged 50-89 years) with cataracts. Of these subjects, 67 completed the study. Subjects were implanted either with a foldable IOL $(n=38)$ or a rigid IOL $(n=29)$. Best corrected Snellen visual acuity and contrast sensitivity (2-28 cycles/degree) were measured approximately 2 months postoperatively. Comparisons were made between the pseudophakic groups and an additional group of 28 age-matched, normal subjects (aged 58-81 years). Non-parametric (Kruskal-Wallis and Mann-Whitney $U$ ) as well as parametric (ANOVA and Pearson) statistics tests were used in the analysis of results.

Results All pseudophakes had corrected visual acuity of $6 / 9$ or better. There were no significant differences in visual acuity (Kruskal-Wallis, $p=0.61$ ) or contrast sensitivity (ANOVA, $p=0.33$ ) between the three groups. The mean residual refractive error of the foldable IOL group was hypermetropic in comparison with the rigid IOL group but not significant at the $99 \%$ level of probability (Mann-Whitney $U, p=0.02$ ). There was no significant difference in astigmatism between the groups.

Conclusions Implanting a foldable acrylic IOL gave no post-operative benefit in visual acuity and contrast sensitivity to pseudophakes over a less expensive rigid PMMA IOL, within this post-operative period. This study did not provide a comparison between the two lenses regarding post-operative complications, in particular the important long-term complication of posterior capsular opacification (PCO). Until and if any particular advantage of a given IOL in reduced incidence of PCO is confirmed, it appears more economical to implant the conventional rigid PMMA IOL using small-incision surgery.

Key words Intraocular lens (foldable, rigid), Pseudophakia, Vision performance

Since the first intraocular lens (IOL) implantation surgery in 1949 by Harold Ridley there have been many improvements in the procedure and the device. Phacoemulsification has allowed dramatic reductions in the size of the incision. The earlier large, typically 10-12 mm, incision required to allow intact removal of the crystalline lens required multiple sutures and frequently caused high levels of induced astigmatism. Following the introduction of phacoemulisification, IOL size became the limiting factor. ${ }^{1}$ This 'small-incision' surgery, with typical incisions of $5.0-5.5 \mathrm{~mm}$, commonly required a single suture. A foldable IOL allows 'micro-incision' surgery, which is limited by the $3 \mathrm{~mm}$ diameter of the phacoemulsification probe, the incision being small enough to be self-sealing. ${ }^{2}$

Induced astigmatism is reduced by decreasing the size of the incision ${ }^{3-5}$ and by making scleral incisions rather than corneal incisions. ${ }^{6-10}$ These previous investigations indicate that there is little induced astigmatism with small or micro-incisions. It also appears that when the incision is small, suturing makes no significant contribution to the magnitude of induced astigmatism. ${ }^{10}$ Hence it would be reasonable to assume that surgery using scleral, self-sealing micro-incisions would minimise induced astigmatism.

As improvement in vision is the major goal of most IOL surgery, visual acuity and contrast sensitivity (CS) are appropriate outcome measures. CS is often considered the best available measure of visual function in daily life. ${ }^{11}$ Various studies have compared vision between IOL designs and materials. For example Skorpik et al. ${ }^{12}$ reported no difference in CS between PMMA and silicone IOLs, while
A.J. Afsar

S. Patel

R.L. Woods

Department Vision Sciences

Glasgow Caledonian

University

Glasgow, UK

S. Patel

Academic Unit of

Ophthalmology

University of Birmingham

Birmingham, UK

W. Wykes

Department of

Ophthalmology

Southern General Hospital

Glasgow, UK

Asfa Afsar MCOptom Department of Vision Sciences

Glasgow Caledonian University

Cowcaddens Road Glasgow G4 OBA, UK

Tel: +44 (0)1413313397

Fax: +44 (0)1413313387

e-mail:ajaf@gcal.ac.uk

This study was reported, in part, at the annual meeting of the American Academy of Optometry, Orlando, Florida, USA, 9 December 1996; at the annual meeting of the Association for Research in Vision and Ophthalmology, Fort Lauderdale, Florida, USA, 13 May 1997; and at the annual meeting of the British Congress of Optometry and Visual Science, City University, London, UK, 11 September 1997

Miss Afsar was supported by a research scholarship provided by the College of Optometrists, London, UK. Alcon Laboratories (UK) supplied the foldable acrylic intraocular lenses to the Southern General Hospital, Glasgow, UK

Proprietary and financial interest: none

Received: 8 October 1998 Accepted in revised form: 29 January 1999 
Lowe and Easty ${ }^{13}$ reported similar visual acuity but better letter CS with polymethyl-methacrylate (PMMA) than HEMA IOLs. Comparing rigid with foldable IOLs Kohnen et al. ${ }^{14}$ found no difference in Snellen visual acuity between the three IOL types, but low-contrast visual acuity with rigid PMMA lenses was better than with foldable silicone IOLs. Re-analysis of their data indicates that there was no difference in low-contrast visual acuity between rigid PMMA and foldable acrylic IOLs. Conversely, Mäntyjärvi and Tuppurainen ${ }^{15}$ reported that silicone IOLs gave better overall CS at 3 cycles/degree, but not at other spatial frequencies, than PMMA IOLs. Whilst these results are slightly contradictory it is apparent that PMMA IOLs gave visual acuity and CS at least equal to several other materials, including silicone. Hence it is not clear that foldable IOLs offer visual benefits above those shown by rigid IOLs.

Good optical properties, dimensional and material stability, and few post-operative complications are desirable properties of any IOL. Since the availability of the first approved foldable silicone IOLs in 1991, ${ }^{2}$ difficulties associated with uncontrollable unfolding characteristics ${ }^{16}$ and discolouration ${ }^{17}$ have been reported along with an incidence of posterior capsular opacification (PCO) higher than reported for PMMA IOLs. ${ }^{18}$ Consequently there is interest in alternatives such as foldable acrylic IOLs.

Since such foldable IOLs are usually more expensive than rigid IOLs, advantages, such as improved vision, accruing from foldable IOL use must be demonstrated to justify their use. Hence, we compared the vision and residual refractive error with a foldable acrylic IOL implanted using micro-incision surgery and a rigid PMMA IOL implanted using small-incision surgery. Also, these groups of pseudophakic subjects were compared with phakic age-matched normal subjects.

\section{Materials and methods}

Eighty-six subjects (aged 50-89 years) with cataracts were recruited prior to IOL implantation from the Southern General Hospital, Ophthalmology Department, Glasgow as part of a 2 year prospective, randomised study. All subjects provided informed consent prior to inclusion in the study, and full ethical approval for the study was granted by the Southern General Hospital Ethics Committee. Subjects were carefully selected to exclude any ocular disease other than the presence of cataract by the operating surgeon (W.W.). A three-piece, foldable, acrylic IOL was implanted in 43 subjects and a one-piece, rigid, PMMA IOL was implanted in 43 subjects.

During post-operative examination, 2 subjects, both with the foldable IOL, were found to have best corrected Snellen visual acuity worse than $6 / 9(20 / 30)$. For both subjects there was evidence of macular drusen that had not been noted during the pre-surgical assessment. It was concluded that these subjects should not have been recruited into the study and hence were not included in the analysis. A further 17 subjects ( 3 from the foldable
IOL group and 14 from the rigid IOL group) were unable to complete the study for reasons unrelated to their cataract surgery. Therefore, data were analysed for 38 pseudophakes with the foldable IOL and 29 with the rigid IOL. A further 28 age-matched subjects (aged 58-81 years) with no evidence of ocular disease and best corrected Snellen visual acuity better than or equal to 6/9 were recruited from Glasgow Caledonian University Eye Clinic as a control group.

The 6/9 Snellen visual acuity limit was chosen for the age-matched group since it was the next line on the available Snellen acuity chart greater than the $95 \%$ confidence limits of the expected normal visual acuity for this age group as described by Elliott et al. ${ }^{19}$

A pre-programmed Storz biometer incorporating the SRK formula was used to calculate IOL power, $P(D)$, required for the desired post-operative refraction: ${ }^{20}$

$$
P=A-2.50 L-0.90 K
$$

where $A$ was the A-constant for a particular IOL and/or manufacturer, $L(\mathrm{~mm})$ was the measured axial length, and $K(\mathrm{D})$ the average Bausch and Lomb keratometer nominal dioptric power. Further modifications to equation (1) were made for $L<22 \mathrm{~mm}$ or $L>24.5 \mathrm{~mm}$ and where ametropia was planned, in accordance with the SRKII formula. ${ }^{20}$ The IOL implanted for each subject was as close as possible to the desired optical power, within the limits of availability $( \pm 0.50 \mathrm{D})$. The desired post-operative equivalent spherical ametropia ranged from -1.3 to $+5.4 \mathrm{D}$. Two types of IOLs were implanted, both manufactured by the same manufacturer: a foldable acrylic IOL or a rigid PMMA IOL.

The foldable IOL was the Alcon Acrysof model MA60BM, which consisted of a $6.0 \mathrm{~mm}$, bi-convex, acrylic optic and modified-C monofilament PMMA haptic. Model MA60BM had an overall IOL length of $13 \mathrm{~mm}$, a suggested A-constant of 118.8 and was available in powers from +10 to $+30 \mathrm{D}$ in $0.50 \mathrm{D}$ steps. The rigid IOL was the Alcon model LX10BD, which consisted of a one-piece bi-convex PMMA design. Model LX10BD had an overall length of $12 \mathrm{~mm}$, with a $5.25 \mathrm{~mm}$ optic, a suggested A-constant of 118.7 and was available in powers from +10 to $+30 \mathrm{D}$ in $0.50 \mathrm{D}$ steps.

All subjects with cataract received monocular phacoemulsification cataract extraction and IOL implantation by one surgeon (W.W.) conducted at the Southern General Hospital, Glasgow. The scleral incision was either 5.5 or $4.0 \mathrm{~mm}$ in length depending on whether the IOL to be implanted was rigid or foldable, respectively. The IOL was implanted in the capsular bag of each subject and the incision was either sealed with one $10^{\circ}$ nylon cross-over suture or left to self-seal. Once the pseudophakic patient had been discharged from the Southern General Hospital Ophthalmology Department, typically following the 1 month routine post-operative examination by the consulting surgeon (W.W.), vision measurements of the best corrected Snellen visual acuity 
and CS were conducted using the post-operative refractive error measured to threshold. This was usually at between 1 and 2 months post-operatively.

This post-operative period was chosen since most patients were discharged from the Southern General Hospital after their 1 month post-operative visit if ocular status was stable. Each patient was advised during this visit to attend for an eye examination with their favoured optometrist for a change in spectacle lenses as required. Although the interval between cataract surgery and the appearance of PCO varies considerably, McDonnell et al. ${ }^{21}$ have reported that PCO usually does not occur until at least 3 months post-operatively, with about $50 \%$ of IOL implant patients developing PCO within 5 years. Since we were interested in the visual outcomes of the surgery unhindered by PCO, the chosen post-operative period should have been a relatively stable period in which eyes had recovered sufficiently from the effects of the surgery and were unlikely to have developed PCO.

Refractive error in the typical sphere, cylinder and axis format is not suitable for relatively simple statistical analyses. Hence we used the Fourier analysis technique described by Thibos et al. ${ }^{22}$ to examine the post-operative refractive error. The three parameters of the spherocylindrical lens can be expressed by a spherical lens of power $M$, and two Jackson cross-cylinders, one at an axis of $0^{\circ}$ with power $J_{0}$, and the other at an axis of $45^{\circ}$ with power $J_{45}$. The 'power vector' ${ }^{22}$ which joins the point $\left(M_{i}\right.$ $\left.J_{\mathrm{o} i} J_{45 i}\right)$ in this three-dimensional dioptric space to the origin is a complete description of the sphero-cylindrical lens. In this dioptric space, conventional statistical methods can be used to evaluate differences between groups. Although the conventional polar plot of cylinder power and axis is no more informative, to aid the reader unfamiliar with the vector form $\left(J_{0}\right.$ and $\left.J_{45}\right)$, we also present the cylindrical correction information in the polar fomat. As $M$ is the equivalent sphere (sphere + cylinder/2), comparison with the calculated IOL power, $P$, and desired refractive error was simple. Residual refractive error was defined as the spherical equivalent difference between the post-operative and desired refractive errors.

Astigmatic error is affected by corneal shape, hence corneal shape changes due to the surgery can induce astigmatism. ${ }^{3-10}$ Since cataract can induce astigmatism, it was not possible to ascertain the presence of surgically induced astigmatism from a difference between preoperative and post-operative refractive error. However, if there was induced corneal distortion, a difference between post-operative astigmatism of the pseudophakes and the astigmatism of the control group would be expected. All incisions were centred at $110^{\circ}$, irrespective of whether they were right or left eyes (i.e. incision approximately from $130^{\circ}$ to $90^{\circ}$ or $125^{\circ}$ to $95^{\circ}$ for small-incision or micro-incision respectively). Therefore, if the surgical procedure had a steepening or flattening effect, the mean power vector of the IOL groups would be expected to have a different orientation and magnitude from that of the control group.
For vision measurement all subjects wore the appropriate optical correction (as determined from the post-operative refractive error) adjusted for the viewing distance. Visual acuity was measured, to threshold, at $6 \mathrm{~m}$ using an internally lit $\left(756.0 \pm 80.8 \mathrm{~cd} / \mathrm{m}^{2}\right)$ Snellen chart. Although visual acuity measured using a Bailey-Lovie chart would have been more precise, ${ }^{23}$ the pre-assessment of the subjects with cataract and part of the post-operative assessment were conducted in a hospital environment where a Snellen chart was the standard.

CS was measured using a computer-based system with a Cambridge VSG2/3 board and an EIZO monitor (Flexscan T560i-T) which displayed sine-wave gratings of $2,4,8,16$ and 28 cycles/degree with a mean luminance of $53 \pm 4 \mathrm{~cd} / \mathrm{m}^{2}$. The stimulus was circular, subtending $1^{\circ}$ at the eye, and was viewed at $2 \mathrm{~m}$ in all cases. Following a practice session, subjects responded to a spatial, two alternate forced choice presentation by indicating on which side of the monitor, left or right, the stimulus was presented. Stimuli were randomly interleaved, with contrast determined by a staircase algorithm. Threshold contrast was defined as the mean of the log contrast at each reversal within the 30 presentations per spatial frequency.

As Snellen visual acuity has unequal intervals and the level of difficulty varies with each line of the chart, nonparametric statistics appropriate for ordinal data were used (Mann-Whitney $U$-test, Kruskal-Wallis one-way analysis of variance). CS data were approximately normally distributed and hence parametric statistics were appropriate (repeated measures analysis of variance: ANOVA). Where refractive correction data were not normally distributed, non-parametric statistics were applied. Though mean age was not significantly different between the three groups (Kruskal-Wallis, $p=0.09$ ), since vision varies with age, age was included

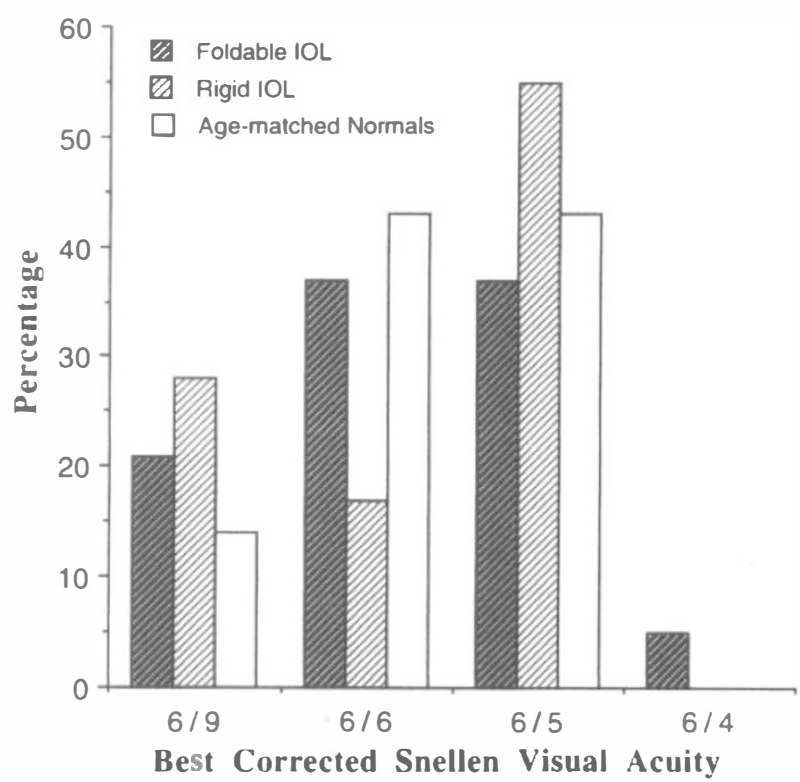

Fig. 1. There was no significant difference in Snellen visual acuity between the three groups (Kruskal-Wallis, $\mathrm{p}=0.61$ ). 


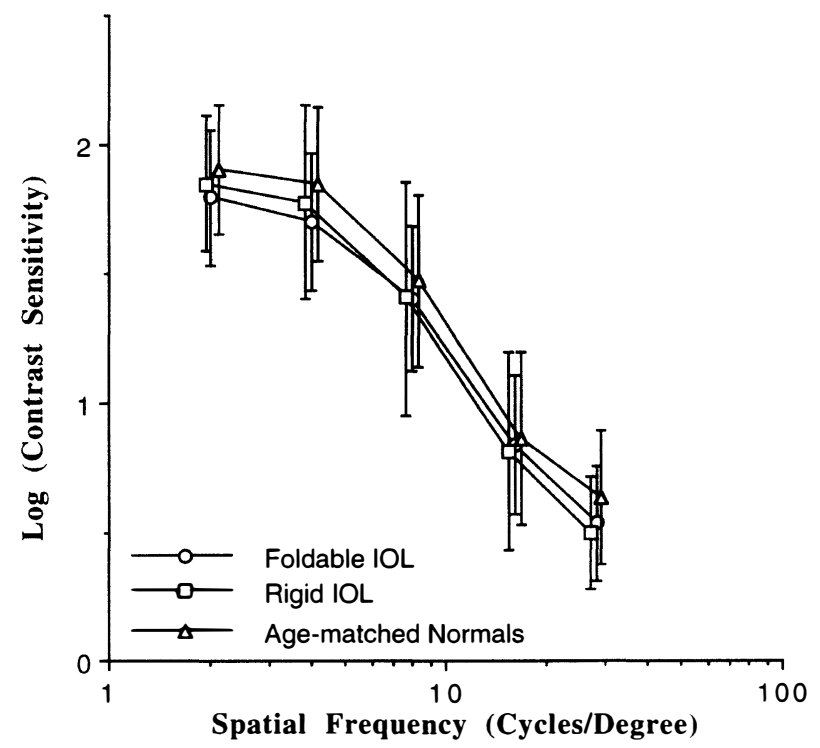

Fig. 2. There was no significant difference in contrast sensitivity between the three groups ( $A N O V A, \mathrm{p}=0.33$ ). The spatial frequencies of points have been staggered to show the error bars (standard deviation) more clearly.

where possible as a co-variate in analyses. A probability level of $\alpha<0.01$ was accepted as significant for all analyses.

\section{Results}

During surgery all subjects with rigid IOLs were sutured as described. In addition, 15 subjects with foldable IOLs were sutured during surgery as deemed necessary by the operating surgeon (W.W.) for ocular health and safety. These 15 subjects had slightly worse best corrected Snellen visual acuity than the remaining 23 subjects with foldable IOLs who had no suture. This finding was not significant at the $99 \%$ level of probability (Mann-Whitney $U, p=0.02)$. Further, the CS of this foldable IOL withsuture group appeared lower than that of the foldable IOL no-suture group (Fig. 2), although there was a lack of statistical significance (ANOVA, $p=0.09$ ) to support this claim. Consequently we pooled all subjects with foldable IOLs as one group of subsequent analyses.

All pseudophakes had a Snellen visual acuity of 6/9 or better (Fig. 1). There was no significant difference in Snellen visual acuity (Kruskal-Wallis test, $p=0.61$ ) and no significant difference in contrast sensitivity (ANOVA, $p=0.33$ ) between the foldable IOL, rigid IOL and control groups.

Most pseudophakes implanted with the foldable IOL had a post-operative refractive error more hypermetropic than the desired refractive error (Table 1). The average
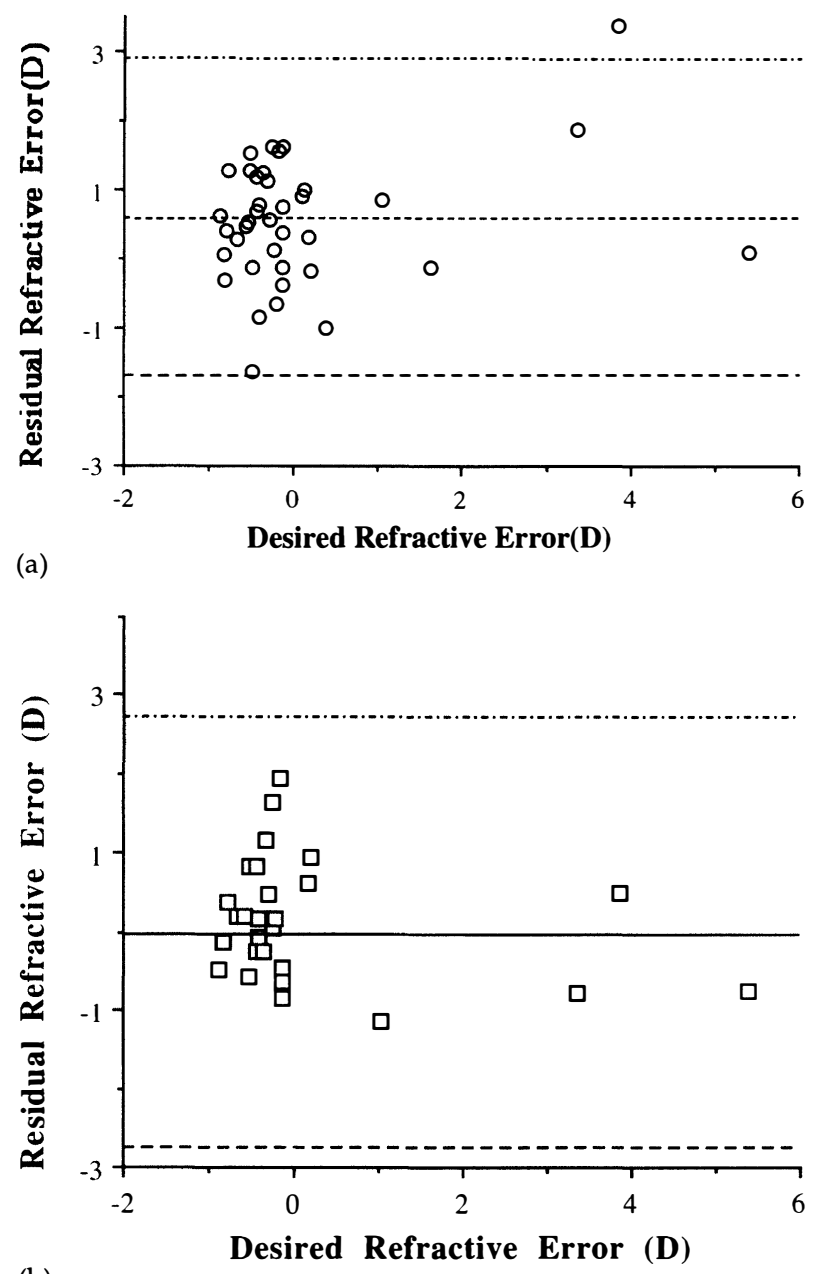

(b)

Fig. 3. Mean residual refractive error of the acrylic foldable IOL group was more hypermetropic than that of the rigid IOL group

(Mann-Whitney $\mathrm{U}, \mathrm{p}=0.02$ ) but not significant at the $99 \%$ level of probability. The ability to achieve the desired post-operative refractive error, as described by the limits of agreement ( $99 \%$ confidence limits) of the residual refractive error, was slightly, but not significantly, better for (a) the acrylic foldable IOL group than (b) the rigid PMMAIOL group. Residual refractive error did not vary with the power of the desired post-operative refractive error for either group.

residual refractive error of pseudophakes implanted with foldable IOLs $(+0.56 \pm 0.93 \mathrm{D})$ was greater than that of those implanted with rigid IOLs $(-0.03 \pm 1.06 \mathrm{D})$, although this was not significant at the $99 \%$ level of probability (Mann-Whitney $U, p=0.02$ ). Given the more hypermetropic residual refractive errors of the foldable IOL groups it is not surprising that fewer subjects in the foldable IOL groups (62\%) had a residual refractive error within the range $\pm 1.00 \mathrm{D}$ than for the rigid IOL group (82\%). Overall $72 \%$ of pseudophakes had a residual refractive error within the range $\pm 1.00 \mathrm{D}$. There was no

Table 1. Means, standard deviation and range of values for the two groups of pseudophakes and the group of age-matched normals

\begin{tabular}{|c|c|c|c|c|c|c|}
\hline & \multicolumn{2}{|c|}{ Foldable IOL } & \multicolumn{2}{|c|}{ Rigid IOL } & \multicolumn{2}{|c|}{ Age-matched normals } \\
\hline & Mean $\pm S D$ & Range & Mean $\pm S D$ & Range & Mean $\pm S D$ & Range \\
\hline Age (years) & $74 \pm 9$ & 50 to 89 & $70 \pm 9$ & 53 to 87 & $69 \pm 6$ & 58 to 81 \\
\hline Desired refractive error (D) & $0.1 \pm 1.3$ & -0.9 to 5.4 & $-0.4 \pm 0.6$ & -1.3 to 1.7 & $\mathrm{~N} / \mathrm{A}$ & $\mathrm{N} / \mathrm{A}$ \\
\hline Post-operative refractive error (D) & $0.6 \pm 1.8$ & -2.1 to 7.3 & $-0.4 \pm 1.2$ & -4.1 to 1.9 & $\mathrm{~N} / \mathrm{A}$ & $\mathrm{N} / \mathrm{A}$ \\
\hline Residual refractive error (D) & $0.6 \pm 0.9$ & -1.6 to 3.4 & $-0.03 \pm 1.1$ & -4.0 to 1.9 & $\mathrm{~N} / \mathrm{A}$ & $\mathrm{N} / \mathrm{A}$ \\
\hline
\end{tabular}




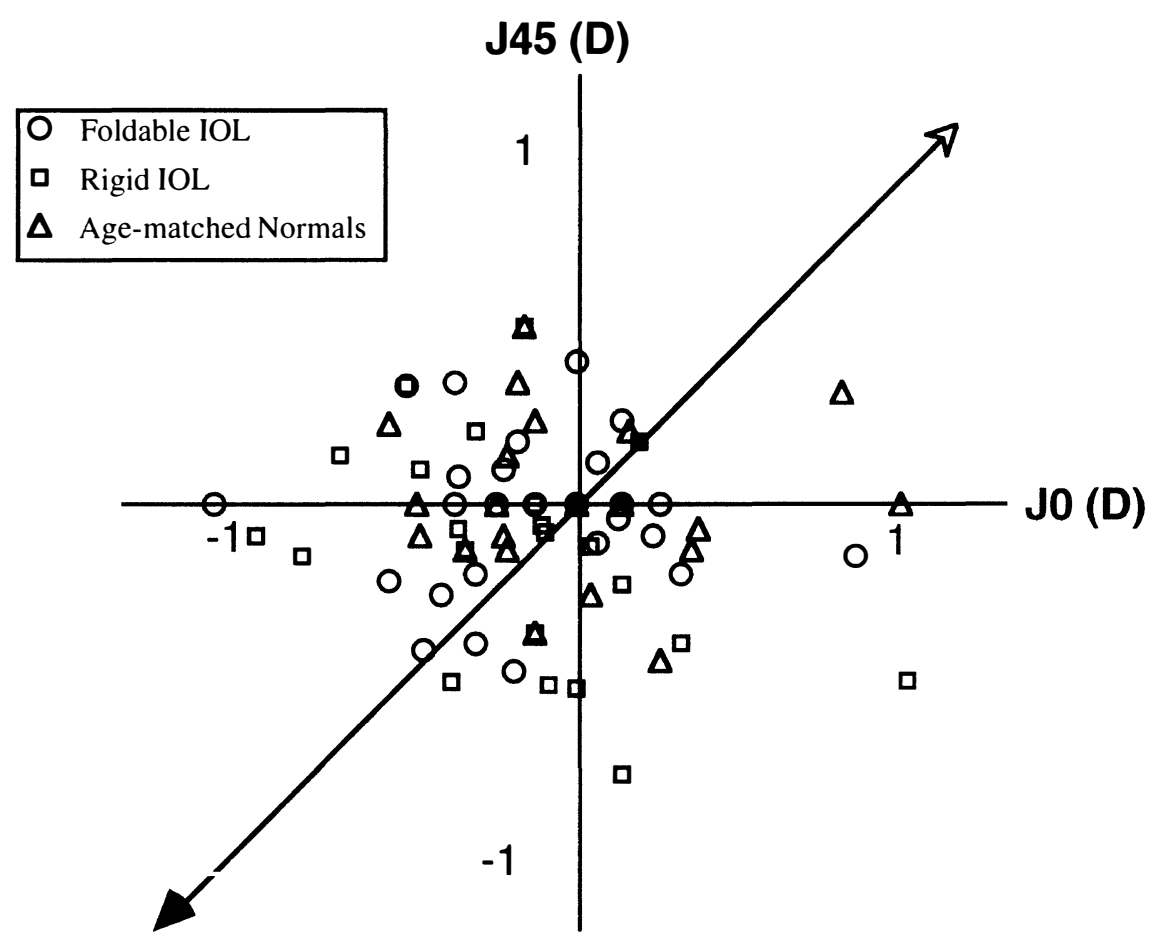

(a)

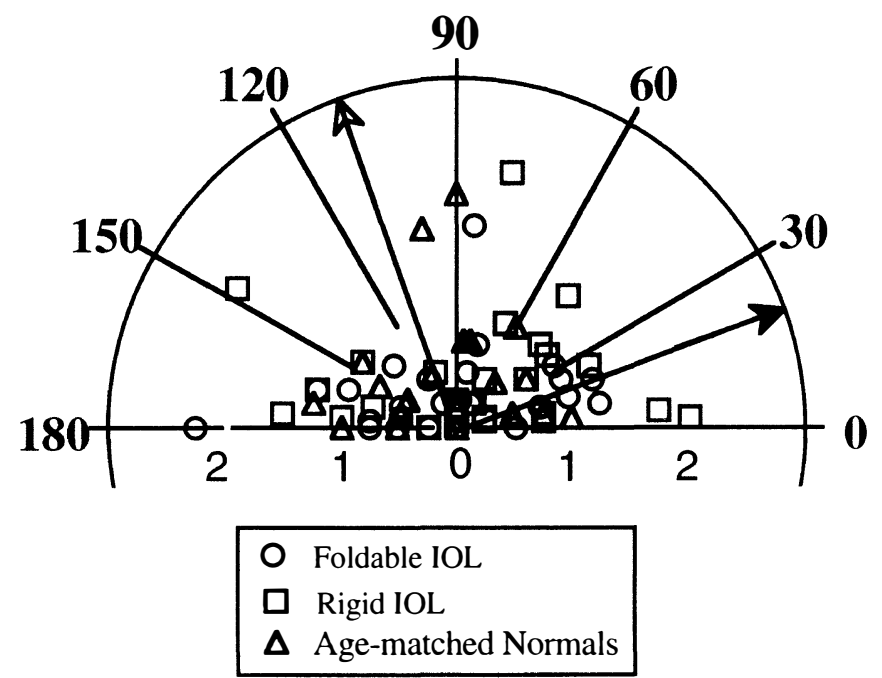

(b)

Fig. 4. (a) Fourier cylindrical components ${ }^{22}$ of the refractive corrections of the three groups. The surgical incision was centred at $110^{\circ}$. For induced astigmatism, the expected mean power vector would lie in the direction of the two arrows (unfilled arrow, 'steepening' effect; filled arrow,

'flattening' effect), if corrected using a negative cylinder. The magnitude of the mean power vector for the age-matched group was found to be not significantly difference from zero $(\mathrm{t}$-test, $\mathrm{p}>0.5)$. The magnitudes of the mean power vectors for the two pseudophakic groups were found to be not significantly different from that of the age-matched mean power vector ( $\mathrm{t}$-test, $\mathrm{p}>0.5$ ). (b) Polar plot of the positive cylinders and axes. The arrows indicate the direction of the mean power vector transformed to the positive cylinder form if there had been any induced astigmatism (unfilled arrow, 'steepening' effect; filled arrow, 'flattening' effect).

significant correlation between the desired and residual refractive errors for any group of pseudophakes (Fig. 3). The $99 \%$ limits of agreement, as described by Bland and Altman $^{24}$ and shown in Fig. 3, were \pm 2.30 (range 2.90 to $-1.70 \mathrm{D})$ for the foldable IOL group, and $\pm 2.74 \mathrm{D}$ (range 2.71 to $-2.77 \mathrm{D}$ ) for the rigid IOL group. There was no significant difference in the limits of agreement between the two groups $(F=1.4, p>0.10)$.

Residual refractive error is a function of the A-constant of the SRK equation (equation 1). As the average residual refractive error of the foldable IOL groups was significantly greater than zero, the
A-constant for the foldable IOL was not correct.

Modification of the A-constant for the individual surgeon (a 'personal' A-constant) has been recommended by Retzlaff et al. ${ }^{20}$ We had used A-constants recommended by the manufacturer. The A-constant appears to have been correct for the rigid IOL, which is reflected in the greater proportion of subjects with the rigid IOL having a post-operative refractive error within $\pm 1.00 \mathrm{D}$.

The relationship between the post-operative $J_{0}$ and $J_{45}$ astigmatic components $^{22}$ of the power vector is illustrated in Fig. 4a. Fig. 4b shows the same data plotted in the conventional polar (positive cylinder) form. There 
was no difference in astigmatism $\left(J_{0}\right.$ and $\left.J_{45}\right)$ between the foldable IOL and control groups. The rigid IOL group had a slightly more negative $J_{0}$ component than the other groups (ANOVA, $p=0.01$ ). The mean vector length $(0.03 \mathrm{D})$ for the age-matched group was found to be not significantly different from zero ( $t$-test, $p>0.5$ ). As the mean power vectors of the rigid and foldable IOL groups (0.17 D and $0.13 \mathrm{D}$ respectively) were not significantly different from zero or from the control group ( $t$-test, $p>0.5)$, there was no significant surgically induced astigmatism.

\section{Discussion}

All subjects had a best corrected Snellen visual acuity of $6 / 9$ or better, a result similar to previous reports. ${ }^{9,14,25}$ Apart from the report by Kohnen et al. ${ }^{14}$ we are not aware of any other study comparing acrylic foldable and rigid PMMA IOLs. Low-contrast visual acuity is typically 0.2-0.3 LogMAR units (2 to 3 lines on a Bailey-Lovie chart) lower than high-contrast visual acuity, and therefore measures vision at slightly lower spatial frequencies. Kohnen et al. ${ }^{14}$ found no difference in lowcontrast visual acuity between the IOL types - a result confirmed and extended by our CS measurements at spatial frequencies of 2-28 cycles/degree.

Residual spherical refractive error is an indication of the accuracy of prediction of the appropriate IOL power. The SRK formula (equation 1) and its modifications $(\mathrm{SRKII})^{20}$ were used to predict the IOL power required for each eye. These formulae were reasonable predictors of the IOL power required for the desired refractive error ( $99 \%$ confidence limits between $\pm 2.30 \mathrm{D}$ and $\pm 2.74 \mathrm{D}$ ). While these limits of agreement, a measure of the predictability of the refractive outcome, were smaller (i.e. better) with the foldable IOL than the rigid IOL (Fig. 3), this difference was not statistically significant. Similarly, as no significant induced astigmatism was found, it appears that both small-incision and micro-incision surgery caused minimal post-operative astigmatism.

Optimal IOL surgery would not reduce the optical quality of the eye. Post-operative vision is an indirect measure of the optical quality of the eye. As there was no difference in visual acuity or contrast sensitivity between the foldable IOL, rigid IOL and control groups, and no apparent corneal distortion, we conclude that both smallincision and micro-incision implantation surgery can achieve this goal. Therefore, there was no apparent visual benefit of implanting an acrylic foldable IOL using micro-incision surgery over implanting a rigid PMMA IOL using small-incision surgery.

If there is no benefit to micro-incision surgery and foldable IOLs, implantation of the more expensive foldable IOL may be an unnecessary expense. However, visual and refractive outcomes may not be the only important measures of cost-effectiveness. Although longterm post-operative complications were not considered for this report, possible long-term benefits of acrylic IOLs have been suggested by recent reports. ${ }^{26,27}$ If acrylic foldable IOLs cause fewer post-operative complications (e.g. PCO) and require less post-surgical care they may be cost-effective. Until these reports are confirmed by further, independent studies, it would appear more economical to implant the conventional rigid PMMA IOL using small-incision surgery, as we have found no additional visual benefits from implanting an acrylic foldable IOL using micro-incision surgery.

\section{References}

1. Kelman CD. Phacoemulsification and aspiration. Am J Ophthalmol 1967;64:23-35.

2. El-Maghraby A, Kraff MC, Raanan MG. Foldable IOL technology: an overview. In: Martin RG, Gills JP, Sanders DR, editors. Foldable intraocular lenses. Thorofare, NJ: Slack, 1993:1-12.

3. Stienert RF, Brint SF, White SM, Fine H. Astigmatism after small incision cataract surgery. Ophthalmology 1991;98:417-23.

4. Werblin TP. Astigmatism after cataract extraction: 6-year follow up of 6.5- and 12-millimetre incisions. J Refract Corneal Surg 1992;8:448-58.

5. Uusitalo RJ, Ruusuvaara P, Järvinen E, et al. Early rehabilitation after small incision cataract surgery. J Refract Corneal Surg 1993;9:67-70.

6. Gross RH, Miller KM. Corneal astigmatism after phacoemulsification and lens implantation through unsutured scleral and corneal tunnel incisions. Am J Ophthalmol 1996;121:57-64.

7. Lyhne N, Corydon LA. Astigmatism after phacoemulsification with adjusted and unadjusted sutured versus sutureless $5.2 \mathrm{~mm}$ superior scleral incisions. J Cataract Refract Surg 1996;22:1206-10.

8. Olsen T, Dam-Johansen M, Bek T, Hjortdal JO. Corneal versus scleral tunnel incision in cataract surgery: a randomised study. J Cataract Refract Surg 1997;23:337-41.

9. Sanchez E, Artaria L. Evaluation of the first 50 ACR 360 acrylic intraocular lens implantations. J Cataract Refract Surg 1996;22s:137-8.

10. Mendivil A. Intraocular lens implantation through 3.2 versus 4.0 mm incisions. J Cataract Refract Surg 1996;22:1461-4.

11. Owsley C, Sloane ME. Contrast sensitivity, acuity, and the perception of 'real-world' targets. Br J Ophthalmol 1987;71:791-6.

12. Skorpik C, Gottlob I, Weghaupt H. Comparison of contrast sensitivity between posterior chamber lenses of silicone and PMMA material. Graefes Arch Clin Exp Ophthalmol 1989;227:413-6.

13. Lowe KJ, Easty DL. A comparison of 141 polymacon (iogel) and 140 poly(methyl methacrylate) intraocular lens implants. Br J Ophthalmol 1991;76:88-90.

14. Kohnen S, Ferrer A, Brauweiler P. Visual function in pseudophakic eyes with poly(methyl methacrylate), silicone, and acrylic intraocular lenses. J Cataract Refract Surg 1996;22s:1303-7.

15. Mäntyjärvi $M$, Tuppurainen K. Contrast sensitivity in patients with silicone intraocular lenses. Ophthalmologica 1997;211:214-8.

16. Masket S. Consultation section. J Cataract Refract Surg 1996;22:159-67.

17. Aronosa D. Intraocular lenses: new materials for smallincision cataract surgery. Bull Acad Nat Med 1995;179:557-67.

18. Milazzo S, Turut P, Artin B, Charlin J. Long-term follow-up of three-piece, looped, silicone intraocular lenses. J Cataract Refract Surg 1996;22:1259-62.

19. Elliott DB, Yang KCH, Whitaker D. Visual acuity changes throughout adulthood in normal, healthy eyes: seeing beyond 6/6. Optom Vis Sci 1995;72:186-91. 
20. Retzlaff J, Sanders D, Kraff M. Lens implant power calculation: a manual for ophthalmologists and biometrists. 3rd ed. Thorofare, NJ: Slack, 1991:1-58.

21. McDonnell PJ, Zarbin MA, Green WR. Posterior capsule opacification in pseudophakic eyes. Ophthalmology 1983;90:1548-53.

22. Thibos LN, Wheeler W, Horner D. Power vectors: an application of Fourier analysis to the description and statistical analysis of refractive error. Optom Vis Sci 1997;74:367-75.

23. Lovie-Kitchen JE. Validity and reliability of visual acuity measurements. Ophthalmic Physiol Opt 1988;8:363-70.
24. Bland JM, Altman DG. Statistical methods for assessing agreement between two methods of clinical measurement. Lancet 1986;I:307-10.

25. Oshika T, Suzuki Y, Kikaki H, Yaguchi S. Two year clinical study of a soft acrylic intraocular lens. J Cataract Refract Surg 1996;I:104-9.

26. Ursell PG, Spalton DJ, Pande MV, Hollick EJ, Barman S, Boyce J, Tilling K. Relationship between intraocular lens biomaterials and posterior capsule opacification. J Cataract Refract Surg 1998;24:352-60.

27. Hollick EJ, Spalton DJ, Ursell PG, Pande MV. Lens epithelial cell regression on the posterior capsule with different intraocular lens materials. Br J Ophthalmol 1998;82:1182-8. 